\title{
Concerning the sum of a countable number of mutually exclusive continua in the plane ${ }^{1}$.
}

By

\author{
R. L. M o o r e (Austin, Texas, U. S. A.).
}

In 1918 Sierpinski 2 ) showed that if the sum of a countably infinite collection of closed point sets is bounded then it is not a continuum. He raised the question whether this theorem remains true if the restriction that the sum should be bounded is removed from the hypothesis. It will be shown, in the present paper, that, for the case where each point set of the collection in question is itself a continuum, this question may be answered in the affirmative. I will first establish some auxiliary theorems.

Theorem 1. If the domains $\left.{ }^{3}\right) D_{1}$ and $D_{2}$ have no point in common and the boundary of $D_{1}$ is a bounded subset of the boundary of $D_{2}$ then the boundary of $D_{1}$ is connected.

Proof. Suppose, on the contrary, that $B_{1}$, the boundary of $D_{1}$, is the sum of two mutually separated ${ }^{4}$ ) point sets $H$ and $K$. Each point of $H$ can be enclosed within a circle which neither contains nor encloses any point of $K$. Since $H$ is closed and bounded it follows, by the Heine-Borel-Lebesgue Theorem, that there exists a finite

1) Remarque de la Rédaction. Cet ouvrage contient des résultats que M. Moore a trouvés indépendammont de M. Mazurkiewicz, qui a obtena les principanx théorèmes de cet ouvrage dans une note du vol. V des Fund. Math. (p. 188-205).

2) W. Si er pins ki, Un théorème sur les continas, Tôhoku Mathematical Journal, vol. 13, $\mathrm{N}^{0} 4$, June, 1918, pp. 300-303.

3) A domain is a connected point set whose complement is closed.

4) Two point-sets $H$ and $K$ are said to be mutually separated if neither of them contains a point or a limit point of the other one. 
set $G$ of circles such that (a) each point of $H$ is within some circle of the set $G,(b)$ no circle of $G$ either contains. or encloses any point of $K$. Let $L$ denote the point set obtained by adding together the interiors of all the circles of the set $G$. Let $P$ denote some point of $H$ and let $L_{p}$ denote the greatest connested subset of $L$ which contains $P$. It is clear that $L_{P}$ is a domain and that its boundary $T$ contains no point of $B_{1}$. Let $Q$ denote some point of $K$. Let $W$ denote that complementary domain of $L_{P}+I$ which contains $Q$. By a theorem of Brouwer's ${ }^{1}$; $B_{3}$ the boundary of $W$ is connected. Both $P$ and $Q$ are limit points both of $D_{1}$ and of $I_{2}$. But $Q$ is in $W^{r}$ and $P$ is without $W$. Hence both $D_{1}$ and $D_{2}$ contain points in $W$ and points without $W$ and, therefore, since they are connected, each of them containsi a point of $B_{B}$, the boundary of $W$. Thus the connected point set $B_{\mathrm{g}}$ contains at least one point of $D_{1}$ and at least one point which does not belong to $D_{1}$. It follows that $B_{3}$ contains a point of $B_{1}$. But $B_{3}$ is a subset of $T$. Hence $T$ contains a point of $B_{1}$. Thus the supposition that $B_{1}$ is not connected leads to a contradiction.

Theorem 2. If the domains $D_{1}$ and $D_{2}$ have no point in common and $B$; the boundary of $D_{1}$, is a bounded sulsct of the boundary of $D_{2}$ and $O$ is any point of $B$, then $B-O$ is connected.

Proof. Suppose, on the contrary, that $B-0$ is the sum of two mutually separated point sets $B_{1}$ and $B_{2}$. By Theorem $1, B$ is con. nected. It follows that $B_{1}+O$ and $B_{2}+O$ are bounded continua with only the point $O$ in common. Furthermore, if $A_{1}$ and $A_{2}$ are points of $D_{1}$ and $D_{2}$ respectively then the sum of the point sets $B_{1}+0$ and $B_{2}+R$ separates $A_{1}$ from $A_{2}$. It follows ${ }^{2}$ ) that either $B_{1}+O$ or $B_{2}+O$ separates $A_{1}$ from $A_{2}$. Suppose that $B_{1}+O$ does and let

1) L. E. J. Brouwer, Beweis des Jorlansenen Kurvonsatzes, Mathematische Annalen, vol. 69 (1910), p. 170.

2) See Theorems $A$ and $B$ on Page 129 of an article by S. Straszowicz, Fundamenta Mathematicae, t. 4. Theso theorems, are referred to by Straszewicz as having been established by Janise wski in an article titlcd: sur les coupurus du plan faitos par des continus, Rrace matem.-fizyozne, tom XXVI, 1913. A proposition which is a logical consecfuenco of theso theorems of Janiszewski's has been recently establisbed by Miss Anna M, M ullikin in her Doctor's dissertation, which, will appear soon in the Transactions of thr American Mathematical Socioty. This paper had gone to the printers before cither Miss Mallikin or I was aware that the proposition liad alrealy been proved. Apjuarently daniszewski's paper is printed. in Polish. 
$K$ denote that complementary domain of $B_{1}+O$ which contains $A_{1}$. Clearly $K$ contains $D_{1}$. But every point of $\vec{B}_{2}$ is a limit point of $D_{1}$. It follows that $K$ contains $B_{2}$. But every point of $B_{2}$ is a limit point of $D_{2}$. Hence $K$ contains at least one point of $D_{2}$. But $D_{2}$ is connected and it contrins no point $B_{1}+O$. It follows that $K$ cuntains $D_{2}$. Therefore $B_{1}+O$ does not separate $A_{1}$ from $A_{2}$. Thus the supposition that Theorem 2 is false leads to a contradiction.

Theorem 3. The outer ${ }^{1}$ ) boundary of a bounded domain is connected and it is not disconnected by the ommision of any one of its points.

Theorem 3 is a consequence of Theorem 2 and either Theorem 1 or the above mentioned theorem of Brouwer.

Theorem 4. If $G$ is a collection of point sets and $M$ is the point set obtained by adding together all the point sets of the collection $G$ and $N$ is the sum of the boundaries of the point sets of the collection $G$ and the point $P$ is a limit point of $M$, then $P$ either belongs to $M+N$ or is a limit point of $N$.

Theorem 5. If $A$ and $B$ are two distinct points and $M$ is a closed point set which is the sum of a countable collection of mutually exclusive closed point sets $M_{1}, M_{2}, M_{3}, \ldots$ no one of which separates $A$ from $B$, then $M$ does not separate $A$ from $B$.

Proof. Suppose, on the contrary, that $M$ does separate $A$ from $B$. Let $G$ denote the sequence of point sets $M_{1}, M_{2}, M_{3}, \ldots$ Let $M_{1,1}$ denote the point set obtained by adding to $M_{1}$ all of its complementary domains which do not contain $A$ and $B$. Let $M_{2}^{*}$. denote the set compused of all those points of $M_{2}$ that do not belong to $M_{1 ; 1}$. Let $M_{2,2}$ denote the point set obtained by adding to $M_{2}^{*}$ all of its complementary domains which do not contain $A$ and $B$. Let $M_{1,2}$ denote the set of all those points of $M_{1,1}$ which do not belong to $M_{2,2}$. Let $M_{3}^{*}$ denote the set of all those points of $M_{3}$ which do not belong to $\lambda I_{1,2}+M_{2,3}$. Let $M_{3,3}$ denote the point set obtained by adding to $M_{3}^{*}$ all of its complementary domains which do not contain $A$ and $B$. For each positive integer $i(1 \bar{\Sigma} i \equiv 2)$ let $M M_{i, 3}$ denote the set of all those points of $M_{i, 2}$ which do not belong to $M_{3,3}$. This process may be continued. Thus we have a sequence $M_{1}^{*}, M_{2}^{*}, M_{3}^{*}, \ldots$ and an array:

1) If $D$ is a bounded domain and $B$ is its boundary and $C$ is a circle which. encloses both $B$ and $D$, then the outer boundary of $D$ is the boundary of that complementary domain of $B$ which contains $C$. 


$$
\begin{aligned}
& M_{1,1}, \\
& M_{1,2}, M_{2,2} \\
& M_{1,3}, M_{2,3}, M M_{3,3} \\
& M_{1,4}, M_{2,4}, M_{3,4}, M_{4,4}
\end{aligned}
$$

such that $M_{1}^{*}=M_{1}$ and such that, for every $n, \lambda I_{n, n}$ is the point set obtained by adding to $I L_{n}^{*}$ all of its complementary domains which do not contain $A$ and $B$ and $M_{n+1}^{*}$ is the set of all those points of $M_{n+1}$ which do not belong to $M_{1, n}+M_{2, n}+\ldots+M_{n, n}$ and, for every $m$ and $n,(m \geqq n), \lambda I_{n, m+1}$ is the set of all those points of $M_{n, n}$ which do not belong to $M_{m+1, m+1}$.

For every $n$ iet $K_{n}$ denote the set of points common to all the point sets of the infinite sequence $M_{n, n}, M_{n, n+1}, M_{n, n+2}, \ldots$ Some of the point sets of the sequence $K_{1}, K_{2}, K_{3}, \ldots$ may be vacuous. But the boundary of that complementary domain of $M$ which contains $A$ is clearly a subset of $K_{1}+K_{2}+K_{3}+\ldots$ It follows that $K_{1}+K_{2}+$ $+K_{3}+\ldots$ separates $A$ from $B$. But the point sets of the sequenee $K_{1}{ }^{n}, K_{2}, K_{3}, \ldots$ are mutually exclusive and clearly they are all closed and no one of them separates space. This contradicts a theorem of Miss Mullikin's ${ }^{1}$ ) to the effect that if $M$ is the sum of a countabl. number of closed, mutually exchusine point sets $M_{1}, M_{z}, M_{\mathrm{g}}$. (no one of which isconnects a plane $S$, then $M$ does not disennnect $S$, The truth of Theorem 5 is therefore established.

Theorem 6. If $A, B$ and $Q$ are three distinct points and $M$ is a closed and bounded point set which is the sum of a countalle collection $G$ of closed point sets no one of which separates $A$ from $B$ and no two of which have, in common, amy point except (), then .II does not scparate $A$ fiom $B$.

Proof. Let $M_{1}, M_{2}, M_{3}, \ldots$ denote the point sets of the cullection $G$. Subject the plane to an inversion ahout $O$ and let $\bar{A}, \bar{B}$, $\bar{M}_{1}, \bar{M}_{1,}, \bar{M}_{2}, \ldots$ respectively denute the imures of $A, B, M, M_{1}, M_{2}$, . under this inversion. Iset $\bar{G}$ denote the scquence of point sets $\bar{M}_{1}$, $\bar{M}_{2}, \ldots$ It is easy to see that no point set of tho sequerce $G$ stpa. rates $\bar{A}$ from $\bar{B}$. But the point sets of this sequence are mutually 
exclusive and, since $\bar{M}$ is closed and bounded, $M$ is closed. Likewise, the point sets of $\bar{G}$ are all closed. It follows, by Theorem 5 , that $\bar{M}$ does not separate $\bar{A}$ from $\bar{B}$. Hence $\bar{M}+O$ does not separate $\bar{A}$ from $\bar{B}$. Hence $M$ does not separate $A$ from $B$.

Theorm 7. There do not exist two bounded domains $D_{1}$ and $D_{2}$ a closed and bounded point set $K$ which does not separate every point of $D_{1}+D_{2}$ from infinity ${ }^{\mathrm{I}}$ ) such that each of the domains $D_{1}$ and $D_{2}$ contains every. point of the boundary of the other one which does not belong $K$.

Proof. Suppose, on the contrary, that there do exist two bounded domains $D_{1}$ and $D_{2}$, with boundaries $B_{1}$ and $B_{2}$ respectively, and a closed and bounded point set $K$ and a point $O$ belonging to $D_{1}+D_{2}$ such that (1) $K$ does not separate $O$ from infinity, and (2) $\left(B_{1}+K\right)-K$ is a subset of $D_{2}$ and $\left(B_{2}+K\right)-K$ is a subset of $D_{1}$. Let $C$ denote a circle which encloses $D_{1}+D_{2}+B_{1}+B_{2}+K$ and let $X$ denote a point on $C$. There exists a simple continuous arc $O X$ which enntains no point of $K$. In the order from $O$ to $X$ on the are $\circlearrowleft X$, there exists a point $Z$ which is the last point that this are has in common with $B_{1}+B_{2}$. Since $Z$ belongs either to $B_{1}$ or to $B_{2}$, it therefore belongs either to $D_{2}$ or to $D_{1}$. Hence, since $D_{1}$ and $D_{2}$ are domains, the boundary of one of them contains a point which lies on $O X$ between $Z$ and $X$. Hence $Z$ is not the last point of $B_{1}+B_{2}$ on the arc $O X$. Thus the supposition that Theorem 7 is false leads to a contradiction.

Theorem 8. If the boundary of a bounded domain $D_{2}$ is a subset of a simply connected bounded domain $D_{1}$ then $D_{\mathbf{2}}$ is itself a subset of $D_{1}$.

Proof Suppose, on the contrary, that $D_{2}$ is not a subset of $D_{1}$. Since the boundary of $D_{2}$ is a subset of $D_{1}$ there is at least one point of $D_{\mathbf{2}}$ in $D_{1}$ ]. Thus $D_{\mathbf{2}}$ contains at least one point of $D_{1}$ and at least one point which does not belong to $D_{1}$. But $D_{2}$ is connected. Hence it contains at least one point of $B_{1}$, the boundary of $D_{1}$. But, since $D_{1}$ is simply connected, $B_{1}$ is connected. Thus the connected point set $B_{1}$ contains a point of $D_{2}$ and therefore, since it contains no puint of $B_{2}$, the boundary of $D_{2}, B_{1}$ must be a subset of $D_{2}$. But, by Theorem 7, this is impossible. Thus the supposition that Theorem 8 is false leads to a contradiction.

1) A bounded and closed point set $K$ is said to separate the point $P$ from intinity if $P$ lies in a bounded complementary domain of $K$. 
Theorem 9. If $D$ is a boundel domain, the boundary of $D$ does not contain two closed subsets $B_{1}$ and $B_{2}$ such that (a) either. $B_{1}$ and $B_{2}$ have no point in common or $\left.B_{1} \times B_{2}{ }^{i}\right)$ is a point set which does not separate $D$ from infinity, $(b)$ each of the point sets $B_{1}$ and $B_{2}$ separates $D$ from infinity.

Proof. Suppose, on the contrary, that there exists a bounded domain (1) whose boundary contuins two such closed subsets $B_{1}$ and $B_{2}$. Let $D_{1}$ and $D_{2}$ denote those complementary dumains of $B_{1}$ and $B_{2}$ respectively which contain $D$. Let $T_{1}$ and $T_{0}$ respectively denote the bounduries of $D_{1}$ and $D_{2}$. Cleurly $T_{1}-T_{1} \times T_{2}^{\prime}$ is a subset of $D_{2}$ and $T_{2}-T_{1} \times T_{2}$ is a subset of $D_{1}$, and $T_{1}^{\prime} \times T_{2}^{\prime}$ does not separate $D$ from infinity. But this is contrary to Theorem 7 .

Theorem 10. There do not exist, in a plane $S$, a bounded continuum. $M$ and point $\delta$ such that $M-O$ is connected and such that $M$ is the sum of a countable collection $G$ of continua $M_{1}, M_{2}, M_{9}, \ldots$ each of which contains $O$, no tu'o of which have, in common, any point except 0 , and no one of which separates the plane $S$ or is itseli disconnected by the omission of the point $O$.

Proof. Suppose, on the contrary, that there exists such a set. Let $C$ denote some definite circle which encloses $M$. A point sot $\lambda U_{n}$ of the set $G$ will be said to be of class 1 if there exists an are $X Y$ which contains only the point $X$ in common with $M$ and only. the point $Y$ in common with $C$, the point $X$ being a point of $M L_{n}$ distinct from $O$. Under these conditions the are $X Y$ will bo said to join $M_{n}$ simply. to $C$. If $a, b, c$ and $d$ are four distinct sots of the collection $G$ and $a$ and $c$ are simply joined to $C$ by the mutually exclusive $\operatorname{arcs} X_{1} Y_{1}$ and $X_{9} Y_{3}$ respectively and also by the mutually exclusive ares $X_{1}^{\prime} Y_{1}^{\prime}$ and $X_{3}^{\prime} Y_{3}^{\prime}$ respectively and, furthermore, of the two domains into which the point set $\left(a+X_{1} Y_{1}+c+X_{3} Y_{9}\right)-$ $-\left(Y_{1}+Y_{a}\right)$ separates $\left.^{2}\right)$ the interior of $C$, one contains $b-O$ and.

1) If $M$ and $N$ are two point sots, with at least ono point in common, then $M \times N$ denotes the sot of all points which are common to $M$ and $N$.

2) Since noither a nor $X_{1} Y_{1}$ soparates the plano $S$ and they have only one point in cornmon, therefore, by a theorem of Janiszewski's meritionod above, their sum docs not separate $S$. Similarly, $c+X_{3} Y_{3}$ loce not separate $S$. Isut $a+x_{1} X_{1}$ and $c+X_{3} Y_{3}$ have only $O$ in common. Henco $a+X_{1} Y_{1}+c+X_{3} Y_{3}$ does not separate $S$. The circle $C$ is the sum of two arcs $Y_{1} A Y_{3}^{*}$ and $Y_{1} B X_{B}$ which havo,

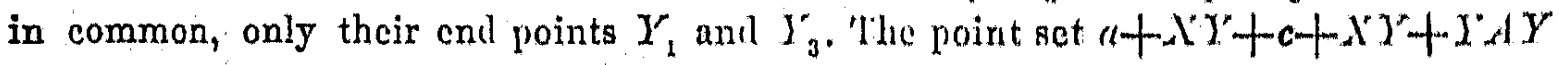
has unly one bounded complementary domain $H_{1}$ and $\left.a+x_{1}\right)_{1}+\lambda_{3} x_{3}+x_{1} \lambda y_{\mathrm{y}}$ has only one bouniled complementary domain $H_{2}$. (Cf, A. Rose nt h a l, Teilung iler 
the other contains $d-O$ and $X_{1}^{\prime} Y_{1}^{\prime}+X_{3}^{\prime} Y_{3}^{\prime}$ has no point in common with $b+d$, then of the two domains into which $\left(a+X_{1}^{\prime} Y_{1}^{\prime}+c+X_{3}^{\prime} Y_{3}^{\prime}\right)-$ $-\left(Y_{1}^{\prime}+Y_{3}^{\prime}\right)$ separates the interior of $C$, one contains $b-O$ and the other contains $d-O$. If $a, b, c$ and $d$ are four distinct point sets of the collection $G$ and there exist two ares $X_{1} Y_{1}$ and $X_{3} Y_{3}$ satisfying, with respect to $a, b, c$ and $d$, the conditions stipulated above, then $a$ and $c$ are said to ordinally separate $b$ from $d^{1}$ ). It can be shown that (1) if $a, b, c$ and $d$ are four distinct point sets of class 1 then two of them ordinally separate the other two from each other und if $a$ and $c$ separate $b$ from $d$ then $b$ and $d$ separate $a$ from $c$, but $a$ and $d$ do not separate $b$ from $c$, (2) if $a$ and $c$ are distinct $G$-sets ${ }^{2}$ ) of class 1 and $b$ is $a G$-set (either of class 1 or not) and there exists some $G$-set which is separated from $b$ by $a$ and $c$ then there exists $a G$-set of elass 1 which is separated from $b$ by $a$ and $c$.

With the aid of the fact that $M-O$ is connected it can be easily proved that there exist four distinct $G$-sets $a_{1}, a_{2}, a_{8}$ and $a_{4}$, all of class 1 and such that $a_{1}$ and $a_{3}$ are separated from each other by $a_{2}$ and $a_{4}$. Let us now confine our attention to the collection $\bar{G}$ consisting of $a_{1}$ and $a_{3}$ together with those $G$-sets which are separated from $a_{4}$ by $a_{1}$ and $a_{3}$. It can easily be seen that if $x$ and $y$ are two distinct $\bar{G}$-sets and $x$ is of class 1 then $y$ is separated either from $a_{1}$ by $x$ and $a_{3}$ or from $a_{8}$ by $x$ and $a_{1}$. In the first case $y$ will be said to follow $x$. In the second case it will be said to precede $x$. It is clear that if $x$ and $y$ are two distinct $\bar{G}$-sets, both of class 1 , then (a) either $x$ follows $y$ or $y$ follows $x$, and (b) if $x$ follows $y$ then $y$ precedes $\left.{ }^{9}\right) x$ and does not follow $x$.

Ebene durch irreduzible Kontinua, Sitzungsber. der Math.-phys. Klasse der Bayerischen Akad. der Wiss. München, 1919, p. 102, Theorem 6. For a more general result see a theorem on p. 130 of the above mentioned paper of Straszewicz. But see also Theorem 5 of Miss Mullikin's dissertation. This resalt of Miss Mullikin's is stated in an abstract on page 349 of The Bulletin of the American Mathematical Society, rol. 27, May 1921). It is easy to prove that if $I$ denotes the interior of the circle $C$, then $l=a+X_{1} Y_{1}+c+X_{3} Y_{3}+H_{1}+H_{2}$.

1) It is to be noted that this does not imply that- $b$ and $d$ ordinally separate $a$ from $c$. The point sets $b$ and $d$ may conceivably not be of class 1 .

2) By a $G$-set is meant a poirt set of the collcction $G$.

3) It is to be observed that no meaning is here attached to the statement that $x$ precedes $y$ in case $y$ is not of class 1 . 
Also if $x, y$ and $z$ are $\overline{G \text {-sets }}$ and $y$ follows $x$ and $x$ follows $z$ then $y$ follows $z$. If $x, y$ and $z$ are $\bar{G}$-sets the statement that $z$ is between $x$ and $y$ means that $(a) x$ and $y$ are of class 1 , and $(b) z$ either follows $x$ and precedes $y$ or follows $y$ and precedes $x$. If $x$ and $y$ are two distinct $\bar{G}$-sets of class 1 then by the segment $x y$ is meant the collection of all those $\bar{G}$-sets which are between $x$ and $y$, while by the interval $x y$ is meant the collection of $\bar{G}$-sets consisting of $x$ and $y$ and all the $\bar{G}$-sets of the segment $x y$. It is easy to see that if $R$ is the point set obtained by adding together all the $\bar{G}$.sets of the segment $x y$, then no point of $R-O$ is a limit point of $M-R$. It is also clear that if a segment, is not vacuous, that is to say if it contains at least one $\vec{G}$-set, then it contains at least one $\bar{G}$-set of class 1. There does not exist more than one pair of distinct $\bar{G}$-sets $(x, y)$ such that $x$ and $y$ are both of class 1 and such that there is no $G$ set between them. For suppose there are two such pairs $(x, y)$ and $(z, w)$. Let $\bar{x}$ and $\bar{y}$ denote $x$ and $y$ respectively or $y$ and $x$ respectively according as $x$ precedes $y$ or $y$ precedes $x$. Let $\bar{z}$ and $\bar{w}$ denote $z$ and $w$ respectively or $w$ and $z$ respectively according as $z$ precedes $w$ or $w$ precedes $z$.

The point sets $\bar{x}$ and $\bar{z}$ must be distinct. For suppose they were identical. Then, since $(\bar{x}, \bar{y})$ and $(\bar{z}, \vec{w})$ are. distinct pairs, either $\vec{y}$ would precede $\bar{w}$ or $\bar{w}$ would precede $\bar{y}$. In the first case $\bar{y}$ would be between $z$ and $w$ and, in the second case, $\bar{w}$ would be between $x$ and $y$, both of which are contrary to hypothesis. Since $\bar{x}$ and $\bar{z}$ are distinct one of them precedes the other one. If $\vec{x}$ precedes $\bar{z}$ let $a$ and $b$ denote $\bar{x}$ and $\bar{y}$ respectively and let $c$ and $d$ denote $\bar{z}$ and $\bar{w}$ respectively. If $\bar{z}$ precedes $\bar{x}$ let $a$ and $b$ denote $\bar{z}$ and $\bar{w}$ respectively and let $c$ and $d$ denote $\bar{x}$ and $\bar{y}$ respectively. Then $b$ either precedes $c$ or is identical with it. For otherwise $c$ would be between $a$ and $b$, contrary to hypothesis. Let $t$ denote either the point set $b$ or the set of points obtained by adding together all the point sets of the interval $b c$, according as $b$ is or is not, identical with $c$. Then the point sets $t-O$ ane $M-t$ are mutually separated and therefore $M-O$ is not connected. Thus the supposition that there exists more than one vacuous segment leads to a contradiction. It follows that there exist two $\bar{G}$-sets $a$ and $b$ of class 1 such that $a$ precedes $b$ and such that if $x$ and $y$ are two $\bar{G}$-sets of class 1 
and belonging to the interval $a b$ then there is a $\bar{G}$-set of class 1 between $x$ and $y$.

Let $e$ denote a $\bar{G}$-set of class 1 which lies between $a$ and $b$. Let $x_{1}$ denote the point set of lowest subscript in the sequence $M M_{1}, M M_{2}, M_{3}, \ldots$ which belongs to the segment ae, is of class 1 and is distinct from $M_{1}$ : The intervals $a x_{1}$ an $e x_{1}$ do not both contain $M_{1}$. If $a x_{1}$ contains $M_{1}$ let $y_{1}$ and $z_{1}$ denote $x_{1}$ and $e$ respectively. If $a x_{1}$ does not contain $M Y_{1}$ let $y_{1}$ and $z_{1}$, denote $a$ and $x_{1}$ respectively. In either case $y_{1}$ precedes $z_{1}$, the interval $y_{1} z_{1}$ is a subset of the interval $a e$ and the interval $y_{1} z_{1}$ does not contain $M_{1}$. Similarly, "the interral $y_{1} z_{1}$ contains two $G$-sets $y_{2}$ and $z_{2}$ of class 1 and such that $y_{2}$ precedes $z_{2}$ and the interval $y_{2} z_{2}$ does not contain $M_{2}$. This process may be continued. Thus there exist two infinite, sequences $y_{1}, y_{2}, y_{3}, \ldots$ and $z_{1}, z_{2}, z_{3}, \ldots$ of $\bar{G}$-sets of elass 1 , all belonging to the interval ae and such that, for every $n$, (1) $y_{n}$ precedes $z_{n}$, (2) $y_{n}$ either precedes, or is identical with, $y_{n+1}$ and $z_{n+1}$ either precedes or is identical with $z_{n}(3)$ the interval $y_{n} z_{n}$ does not contain $M_{2}$. In a similar way it may be shown that there exist two sequences $u_{1}, u_{2}, u_{3}, \ldots$ and $v_{1}, v_{4}, v_{8}, \ldots$ of $\bar{G}$-sets of class 1 , all belonging to the interval $e b$ and such that, for every $n,(1)$ $u_{n}$ precedes $v_{n}$, (2) $u_{n}$ either precedes or is identical with $u_{n+1}$ and $v_{n+1}$ either precedes or is identical with $v_{n},(3)$ the interval $u_{n} v_{n}$ does not contain $M r_{n}$.

Let $L$ denote the point set obtained by adding together all the intervals $z_{1} u_{1}, z_{2} u_{2}, z_{3} u_{3}, \ldots$ The point sets $L-O$ and $L-M$ are mutually separated and therefore $M-O$ is not connected. Thus the supposition that Theorem 10 is false led to a contradiction.

Theorem 11. There does not exist, in a plane $S$, an unbounded continuum which is the sim of a countable collection $G$ of mutually exclusive continua $M_{1}, M_{2}, M_{3}, \ldots$

Proof. Suppose, on the contrary, that there does exist "such a continuum $M$. Each point set of the collection $G$ is unbounded. For suppose that one of them (call it $M_{i}$ ) is bounded. Then there exists a cirle $J$ which encloses $M_{1}$. Let $X$ denote a point of $M_{1}$. Since the continuum $M I$ contains the point $X$ within $J$ and also contains a point without $J$, therefore $M$ contains a sub-continuum $\bar{M}$ which contains $X$ and at least one point on $J$, but no point without $J$. Since $\bar{M}$ contains a point on $J$ it is not a subset of $M_{i}$ 
Let $M_{i_{1}}, M_{i_{4}}, M_{i_{8}}, \ldots$ denote those point sets of the collection $G$ which have points in common with $\bar{M}$. For each $n$, let $\bar{M}_{i_{n}}$ denote the set of points common to $\bar{M}$ and $M_{i_{n}}$. Since $\bar{M}$ and $M_{i_{n}}$ are closed so is $\bar{M}_{i_{n}}$. Thus the bounded continuum $\bar{M}$ is the sum of the countable collection of closed point sets $\bar{M}_{i_{1}}, \bar{M}_{i_{2}}, \bar{M}_{i_{3}}, \ldots$ But this is impossible according to Sierpiński's theorem. Thus the supposition that the point sets of the collection $G$ are not all unbounded leads to a contradiction. Furthermore the set $M$ does not fill up the whole plane. For suppose that it does. Let $X$ denote a point of $M_{1}$ and let $Y$ denote. a point of $M_{\mathbf{2}}$. Then the straight line interval $X Y$ is a bounded continuum which is the sum of a countable collection of closed point sets $\bar{M}_{h}, \bar{M}_{i_{i}}, \bar{M}_{i,}, \ldots$ where, for every $n, \bar{M}_{i_{n}}$ is the set of points common to the interval $X Y$ and the point set $M_{n_{n}}$. Thus again a contradiction is reached. Hence there exists, in plane $S$, a point $O$ which does not belong to $M$. Let the plane $S$ be subjected to an inversion about some circle with center at $O$. Let $T$ denote the image of $M$ and, for every $n$, let $T_{n}$ denote the image of $M_{n}$, under this inversion. Let $K$ denote $T+Q$ and, for every $n$, let $K_{n}$ denote $T_{n}+O$. Let $\bar{G}$ denote the collection of point sets $K_{1}$, $K_{2}, K_{8}, \ldots$ Clearly $K-O$ is connected and, for every $n, K_{n}-O$ is connected. Now let $c$ denote a circle which encloses $K$ and has its center at $O$. One complementary domain of the point set $K$ contains the circle $c$. Call this domain $D_{0}$. If any point set $K_{n}$ of the collection $\bar{G}$ contains a subset $B_{n}$ which is the complete boundary of any domain $d$ (other than $D_{\mathrm{a}}$ ) which is complementary to $K$ then add all such domains $d$ to the point set $K_{n}$ and let $K_{n}^{*}$ denote the point set constituted by the sum so obtained. In case $K_{n}$ contains no such subset $B_{n}$ then let $K_{n}^{*}$ denote $K_{n}$. We now have a collection $G^{*}$ of point sets $K_{1}^{*}, K_{2}^{*}, K_{3}^{*}, \ldots$ each of which either is identical $_{n}$ with, or contains, some point set of the collection $\bar{G}$. It is clear that the point sets of the collection $G^{*}$ are all continua, that every two of them have in common only the point $O$, that their sum $K^{*}$ is a bounded continuum lying wholly within the circle $c$ and finally that $K^{*}-O$ is connected and, for every $n, K_{n}^{*}-O$ is connected. No domain, except $D_{0}$, which is complemerttury to $K^{*}$ has, for its complete boundary, a subset of any one point set of the collection $G^{*}$. It is conceivable however that there may be a domain, other than $D_{c}$, which is complementary to some point 
set of the collection $G^{*}$ (and which is therefore bounded by a subset of that point set). If such a domain exists it must contain points of $K^{*}$.

Case 1. Suppose there is no domain, other than $D_{c}$, which is complementary to $K^{*}$. Suppose a point set $K_{n}^{*}$ of the collection $G^{*}$ separates the plane. Then $K_{n}^{*}$ has a complementary domain $H$ which contains no point of $D_{c}$. The domain $H$ must be a subset of $K^{*}$. Let $X$ denote a point of $H$. There exists a simple continuous arc $X Y$ which does contain $O$ but lies wholly in $H$ except for the point $Y$ which lies on the boundary of $H$. Every point of the bounded continuum $X Y$ belongs to some point set of the countable collection $G^{*}$ and $X Y$ contains the point $Y$ of $K_{n}^{*}$ and a point $X$ which does not belong to $K_{n}^{*}$. This is contrary to Sierpinski's theorem. Thus the supposition that the plane is separated by any point set of the collection $G^{*}$ leads to a contradiction. Hence, in Case $1, G^{*}$ is a countable collection of continua $K_{1}^{*}, K_{2}^{*}, K_{\mathbf{3}}^{*}, \ldots$ such that $(\alpha)$ their sum $K^{*}$ is a bounded continuum and $K^{*}-O$ is connected, $(b)$ no two of them have, in common, any point except $O,(c)$ no one of them is disconnected by the omission of $O,(d)$ no one of them separates the plane. But this is contrary to Theorem 10. Thus in Case 1 we have a contradiction.

C a s e 2. Suppose now that there exists a domain $D^{*}$, other than $D_{e}$, which is complementary to $K^{*}$. The boundary $k$ of this domain is not a subset of any one point set of the collection $G^{*}$. It follows that $k$ is the sum of a collection $g$ of two or more distinct closed point sets $k_{1}, k_{g}, \ldots$ each of which is a subset of some point set of the collection $\bar{G}$ and no two of which belong to the same point set of this collection. If $k_{n}$ is a point set of the collection $g$, one complementary domain $d_{n}$ of the point set $k_{n}$ contains the domain $D^{*}$. By Theorem 9 there does not exist more than one $n$ such that $d_{n}$ fails to contain $c$. By Theorem 6 there does exist one such $n$. Call it $\bar{n}$. For no $n$ does any complemenlary domain of $k_{n}$, except $d_{n}$, contain a point of $k$. The point set $k$ is a continuum since it is the boundary of a complementary domain of the continuum $K^{*}$. That $k$ contains $O$ and that every $k_{n}$ contains $O$ can be shown by a method similar to that used above to show that every point set of the collection $G$ is unbounded. The point set $k-O$ is connected. For suppose that it is not. Let $w$ denote the outer boundary of $D^{*}$. Then $k-O$ is the sum of two mutually separated point sets $k^{\prime}$ and $k^{\prime \prime}$, 
where $k^{\prime}$ contains at least one point of $w-0$. It is clear that $w$ is a subset of $k_{n}$. By Theorem 3, the point set $w-O$ is connected. Hence $w-O$ is a subset of $k^{\prime}$. The point set $w$ is ${ }^{1}$ ) the complete boundary of at least two domains $D_{1}$ and $D_{2}$ where $D_{1}$ contains $a$ and is unbounded while $D_{2}$ contains $D^{*}$ and is bounded. The domain $D_{2}$ contains every point of $k-0$, which does not belong to $w$. If it contains any points of $K$ which do not belong to $k$, let $Z$ denote the set of all such points. If $X$ is a point of $Z$ then $X$ belongs to a complementary domain of $k$. Call this domain $d_{x}$ and call its boundary $k_{x}$. Then $k_{x}$ is a subset of $k^{2}$. By a theorem of Brouwer's mentioned above, $k_{x}$ is connected and, by Theorem 2, if $k_{x}$ contains $O$ then $k_{x}-O$ is connected. Hence, in any case, the point set $\left(k_{x}+O\right)-O$ is a subset either of $k^{\prime}$ or of $k^{\prime \prime}$. Now let $I^{\prime}$ denote the point set obtained by adding together $k^{\prime}$, all points of $K-0$, if there are any, which do not bolong to $w+D_{2}$, and all points $X$ of $Z$ such that $\left(k_{X}+O\right)-O$ is a subset of $k^{\prime}$. Let $K^{\prime \prime}$ denote the set of points obtained by adding together $k^{\prime \prime}$ and all points $X$ of $Z$ such that $\left(k_{x}+0\right)-0$ is a subset of $k^{\prime \prime}$. With the help of Theorem 4 and the fact that $k^{\prime}$ and $k^{\prime \prime}$ are mutually. se. parated. it is easy to prove that $K^{\prime}$ and $K^{\prime \prime}$ are mutually separated. But clearly $K^{\prime}+K^{\prime \prime \prime}=K-O$. Hence $K-O$ is not connected. Thus the supposition that $k-0$ ) is not connected has led to a contradiction. Since $k-O$ is connected it easily follows that there are infinitely many point sets in the collection $g$. Thus $k_{1}, k_{2}, \ldots$ is an infinite sequence.

If, for any $n, k_{n}$ has a complementary domain other than $d_{n}$ then let $k_{n}^{\prime \prime}$ denote the point set obtained by adding to $k_{n}$ all such complementary domains. If $k_{n}$ has no complementary domain except $d_{n}$, let $k_{n}^{*}$ denote $k_{n}$. Let $g^{*}$ denote the countable collection of point sets $k_{1}, k_{2}, k_{3}, \ldots$ and let $k^{*}$ denote their sum. The point sets of the collection $g^{*}$ are all closed, no two of them have, in common, any point except $O$, their sum $k^{*}$ is closed and connected and, with the aid of the fact that $k-O$ is connected, it can easily be seen that $k^{*}-O$ is connected. Furthermore, no point set of the collection $g^{*}$ separates the plane.

If a point $P$ is separated from $D^{*}$ by a point set $k_{w}$ of the

1) Cf. my paper Concerning continuous curves in the plane Mathematische Zeitschrift, vol. 15 (192\%), pp. 254-260. 
collection $g$ and $P$ belongs to a point set of the collection $G$ which contains some point set of the collection $g$, then $P$ belongs to that point set of the collection $G$ which contains $k_{m}$. For suppose that $K_{n}$ is that point set of the collection $G$ which contains $\dot{k}_{m}$ and suppose that $P$ belougs to $K_{j}$, where $j \neq n$. Let $V$ denote that complementary domain of $k_{m}$ to which $P$ belongs. Suppose first that $K_{j}-O$ is not a subset of $V$. Then clearly $K_{j}-O$ is the sum of the mutually separated sets $L_{1}$ and $L_{2}$, where $L_{1}$ consists of all those points of $K_{j}-O$ which belong to $V$ and $L_{2}$ consists of all those which do not belong to $V$. Hence $K_{j}-O$ is not connected. Thus, in case $K_{j}-O$ is not a subset of $V$, a contradiction is reached. Suppose secoridly that $K_{j}^{r}-O$ is a subset of $V$. Then, since by hypothesis $K_{j}$ contains some point set of the collection $g$, therefore $V$ contains a point $Y$ which belongs to $h$. But $Y$ is a limit point of $D^{*}$. Hence $V$ contains a point of $D^{*}$, contrary to the supposition that $k_{m}$ separates $P$ from $D^{*}$. Thus again a contradiction is reached. It follows that $P$ belongs to $K_{n}$. With the help of this fact it can be shown that, for every $n, k_{i n}^{m}-O$ is connected. For suppose, on the contrary, that there exists a positive integer $m$ such that $k_{m}^{*}-O$ is the sum of two mutually separated point sets $H_{1}$ and $H_{2}$. Let $K_{n}$ denote that point set of the collection $G$ which contains $k_{m}$. If $B$ is the boundary of a complementary domain of $k_{m}$ which contains no point of $D^{*}$, then, by Theorems 1 and $2,(B+O)-O$ is connected. Hence $(B+O)-O$ is a subset either of $H_{1}$ or of $H_{2}$. For each $i(1 \equiv i \equiv 2)$ let $H_{i}^{*}$ denote the point set obtained by adding to $H_{i}$ every point of $K_{n}-O$ which lies in a complementary domain of $k_{m}$ which contains no point of $D^{*}$ and whose boundary is, except for the point $O$, a subset of $H_{i}$. Every point of $K_{n}-O$ belongs either to $H_{1}^{*}$ or to $H_{\tilde{2}}^{\ddot{*}}$. For suppose this is not the case. Then $d_{m}$, that complementary domain of $k_{m}$ which contains $D^{*}$, contains a point $P$ belonging to $K_{n}-O$. Since the point $P$ belongs neither to $D^{*}$ nor to its boundary $k$, it is separated from $D^{*}$ by $k$. It follows, with the help of Theorem 6 , that $P$ is separated from $D^{*}$ by some point set $k_{t}$ of the collection $g$. Clearly $k_{t}$ must be distinct from $k_{m}$. 'But it has been shown above that this is impossible. It follows that $K_{n}-O$ is the sum of the two point sets $H_{1}^{*}$ and $H_{2}^{\ddot{*}}$. But clearly these tiwo point sets are mutually separated. Hence $K_{n}-O$ is not connected. Thus the supposition that $k_{m}^{*}-O$ is not connected has led to a contradiction. 
202 R. L. Moore: Concerning the sum of continua.

Now let $A$ denote some definite point of $D^{*}$ and subject the plane $S$ to an inversion about the point $A$. Let $\bar{k}$ and $\bar{k}_{\bar{n}}$ denote the point sets obtained by adding $A$ to the images of $k^{*}$ and $k_{n}^{*}$ respectively and, for every $n$, except $\bar{n}$, let $\bar{k}_{n}$ denote the image of $k_{n}^{*}$ ard let $\bar{O}$ denote the image of $O$, under this inversion. Then $\bar{k}$ is a bounded continuum, $\bar{k}-\bar{O}$ is connected, and $\bar{k}$ is the sum of the countable collection of continua $\overline{k_{1}}, \bar{k}_{2}, \ldots$ Furthermore, every one of these continua contains $\bar{O}$, no two of them have in common, any point except $\bar{O}$, no one of them separates the plane $S$ and no one of them is disconnected by the omission of the point $O$, 'But this is contrary to Theorem 10. Thus in Case 2 we have a coutradiction.

The supposition that Theorem 11 is false has led to a contradiction, both in Case 1 and in Case 2. The truth of this theorem is therefore established. 